[Draft version - please do not cite. Final version can be found in 'Studies in History and

Philosophy of Science', 47 (2014), 51-59]

\title{
Is Complexity a Scientific Concept?
}

Paul Taborsky

\begin{abstract}
Complexity science has proliferated across academic domains in recent years. A question arises as to whether any useful sense of 'generalized complexity' can be abstracted from the various versions of complexity to be found in the literature, and whether it could prove fruitful in a scientific sense. Most attempts at defining complexity center around two kinds of notions: Structural, and temporal or dynamic. Neither of these is able to provide a foundation for the intuitive or generalized notion when taken separately; structure is often a derivative notion, dependent on prior notions of complexity, and dynamic notions such as entropy are often indefinable. The philosophical notion of process may throw light on the tensions and contradictions within complexity. Robustness, for instance, a key quality of complexity, is quite naturally understood within a process-theoretical framework. Understanding complexity as process also helps one align complexity science with holistically oriented predecessors such as General System Theory, while allowing for the reductionist perspective of complexity. These results, however, have the further implication that it may be futile to search for general laws of complexity, or to hope that investigations of complex objects in one domain may throw light on complexity in unrelated domains.
\end{abstract}

\section{Table of Contents}

1. Introduction 2

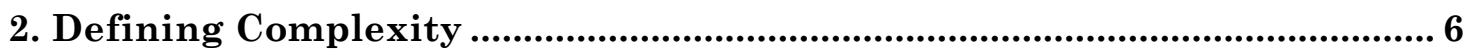

3. Complexity, Structure, and Entropy.................................................................... 9

4. Complexity Every where.................................................................................. 16

5. Complexity, Robustness, and Process …………........................................... 20

6. Concluding Remarks - Complexity, Process, and Anti-reductionism. 27 
Some will still feel the need to account for the impression that something has increased in the history of the Metazoa ... but if we take a skeptical approach, it seems reasonable to ask at this point - having investigated four kinds of complexity whether complexity has anything to do with the apparent trend ... the possibility should be considered that the appearance of a trend of any sort is an illusion, a trick of the imagination .... thus, at least at present, given the state of our knowledge, we would seem to have little basis for concluding that the "something" is complexity.

— D. McShea, Complexity in Evolution: A Skeptical Assessment

The central character of scientific revolutions is that they alter the knowledge of nature that is intrinsic to the language itself and that is thus prior to anything quite describable as description or generalization, scientific or everyday.

- T. Kuhn, What are scientific revolutions?

\section{Introduction}

Recent years have seen an enormous proliferation of studies concerning complexity and complex systems. The study of complexity and complex phenomena extends from computation, to biology, physics, sociology, economics and even management studies. What, if anything, ties all these diverse areas together? Is there a useful sense of complexity that can be extracted from various disciplinary senses? Is complexity science, or rather, the interdisciplinary behemoth that might be called 'generalized complexity science' (whose institutional incarnation is the well-known Santa Fe Institute), scientifically fruitful, or is it rather beset by a fundamental uncertainty that would belie any such attempts?

In a comparatively early attempt at formulating a characterization of the 'diverse kinds of complex systems' Simon (1962) concluded that such an attempt might not be in vain, and that hierarchy could in fact be the basic key to generalized complexity. As well, he conjectured that descriptions of complex objects are best handled by a mixture of state 
and process descriptions (i.e. descriptions of actions such as those involved in constructing a geometrical object, or in describing metabolism). As state descriptions are conventional in science, his requirement implies that we need to expand our repertoire of scientific terminology by adopting process descriptions in order to best account for the complexity we find in nature. Though tentative and self-confessedly explorative, Simon's conclusions seem to be these: Both process and state descriptions are necessary for understanding complexity; process descriptions have been fundamental for the development of modern science, especially the sciences modeled by differential equations; and hierarchy is fundamental to complexity. Simon's conclusions are stimulating and suggestive, and I believe that he is correct in his focus on process descriptions as an important factor in adequate accounts of complexity. Nevertheless, I suggest his conclusions can be strengthened in the following way:

a) Process is fundamental, not simply an alternative or supplementary source of description. The idea that process is fundamental is a key tenet of process philosophy, which subscribes to the notion that all state descriptions can be replaced by process descriptions, or, better put, that process descriptions capture something fundamental about reality that state descriptions do not.

b) Contrary to Simon, differential equations have little to do with process; classical physics, which uses differential equations for its mathematical 
models, is best understood from the 'substantialist', as opposed to processual viewpoint. ${ }^{1}$

c) There is a possible relationship between process and hierarchy ${ }^{2}$. I offer the following sketch of an argument: A process, (conceived of in process-philosophical terms), is in fact best thought of as a hierarchy. A process possesses unity, temporal coherence, and formal patterning (Rescher, 2000, p. 24). Thus, the stages of a process (every process is made up of stages) cannot be entirely independent elements, due to the formal unity of the process: Processes are characterized by 'interactive relatedness' (Rescher, 1996, p.35). This, combined with the forward orientation of most processes (see p. 10 below), introduces an element of subordination that turns such stages into levels. And reciprocally, the kinds of hierarchy dealt with in complexity usually have a temporal or at least interactive or variational element that renders them amenable to process analysis.

Thus, I would like to put forward the suggestion that generalized complexity is in fact not an incoherent hodge-podge of notions, but is centered around the notion of process; however, not Simon's descriptive notion of process, but the much more

\footnotetext{
${ }^{1}$ See e.g. Rescher (1996), pp. 95, 96 and Riche (2004) pp. 183-189.

${ }^{2}$ As Rescher (1996), p. 54, puts it, "Processes generally consist of processes ... process theorists often use organismic analogies to indicate this idea of different levels of units: Smaller, subordinate (or subsidiary) processes unite to form larger, superordinate (or supersidiary) process-units ...the idea of a hierarchic assemblage of micro- into macro-units is a pervasive and characteristic aspect of process philosophy".
} 
fundamental ${ }^{3}$ idea of process as an ontological category, the process, that is, of process philosophy, not process description. The reasons for placing process, (not simply process description, but process philosophy), at the center of complexity will become apparent in the course of my argument ${ }^{4}$.

The question of the adequacy or validity of this characterization of generalized complexity is a separate issue, however. It is one thing to claim that process can unite the various intuitions or notions behind complexity. It is quite another to claim that such a unified notion can yield scientific results. As McShea (1991) writes (concerning the widespread intuition of a positive correlation between complexity and evolution),

The gestalt of increasing complexity that emerges from an overview of the history of life is an unwavering one. We know, however, that gestalts, even steady ones, can mislead, and thus a study of its possible sources in perceptual or cultural biases seems worthwhile. (p.320)

My conclusion that generalized complexity is best characterized as process is intended more as a diagnosis of the contemporary gestalt, than as a suggestion or recommendation for scientific methodology. Like McShea, I am skeptical of the fruitfulness of the notion of generalized complexity, and certainly do not propose that the

\footnotetext{
${ }^{3}$ Fundamental, because, unlike process description, process as an ontological or philosophical category is incompatible with state description. Process, as a fundamental category, also has very different identity criteria from substance, as will be discussed in section five. No such difference separates state and process descriptions (indeed, the process philosopher would likely claim that Simon's distinction is really a distinction between state and action, not state and process.) ${ }^{4}$ Process is a much better understood and well-defined notion than many other concepts that are often put forth in similar attempts to get a philosophical grip on complexity, such as (but not limited to): Anti-reductionism, post-modernism, non-linearity, and anti-mechanism. Positive reasons for my claim will be offered in what follows.
} 
concept of complexity, whether specific or general, be replaced by that of process. Yet I would like to avoid the suggestion that generalized complexity is an incoherent mess of intuitions - quite the contrary, there is a unity behind the diversity of directions in generalized complexity. At the same time, I doubt that such a unified concept can be scientifically fruitful, and for reasons that can be summed up in the following: McShea, in his assessment of these developments, employs the Kuhnian notion of gestalt to characterize the contemporary picture of complexity. The difference between process philosophy and what process philosophers call 'substantialist' philosophies is a difference similar to the difference between incommensurable Kuhnian paradigms. If generalized complexity is in fact part of a Kuhnian-type gestalt, a gestalt, moreover, grounded not simply in a particular picture or image of particular phenomenon (such as constrained or unconstrained motion, to mention a well-known example from Kuhn) but in a philosophical dichotomy, then the difficulties for complexity science in providing a coherent scientific picture of reality are profound ${ }^{5}$.

\section{Defining Complexity}

Let us make a fresh start. To begin with, there is the intractable problem of defining complexity. Biological complexity, on the one hand, is fairly well understood ${ }^{6}$.

\footnotetext{
${ }^{5}$ A fundamental assumption here is that such Kuhnian-like paradigms or gestalts are not only incommensurable, but of equal scientific value, and so cannot in themselves contribute to scientific knowledge. On this assumption (and the question of whether Kuhn would have held it is still an open one. See e.g. Worrall (2003)), the processual and substantialist viewpoints are equally 'correct', a point likely to be contested by both process and substantialist philosophers, among others. It is my suggestion that much of the frustration surrounding the attempts to characterize generalized complexity can be taken as evidence for the plausibility of this assumption, at least in the particular case of complexity.

${ }^{6}$ I would like to thank an anonymous referee for pressing this point.
} 
There appears to be something of a consensus that complexity in biology is best approached in terms of morphological complexity, that is, number and differentiation of parts and irregularity of their arrangement ${ }^{7}$. This kind of definition is evidently a structural definition, and it is clear that structure must play some part in any approach to complexity ${ }^{8}$. Venturing further afield, into other disciplines, there is much less clarity. Most attempts at explaining complexity to be found in the literature appear to be little more than an employment of metaphors that cycle through a range of equally ill-defined concepts, hardly else than semantic substitutions, shifting explanatory weight from complexity toward sibling notions such as non-linearity, hierarchy, multiplicity, and the like, all of which are barely defined any more adequately. As a start, however, it is to be noted that hierarchy, multiplicity, and non-linearity are clearly structural notions, as is morphological differentiation.

On the other hand, we don't have to look far to see that other classes of notions are often involved in defining complexity — in particular, generative, dynamic and regulatory notions, such as self-organization, emergence, adaptation, and feedback (and we will see later in the discussion that temporal metaphors may be in fact much closer to the heart of complexity than might be apparent initially). But these latter terms are hardly clearer, and many cases are clearly to be understood in some sort of non-literal or nontechnical sense. In these latter senses, such concepts often do not function analytically. For example, how can feedback be distinguished from simple causal interaction? Is the presence of feedback in a system a sign of complexity, or does the interpretation of causal

\footnotetext{
${ }^{7}$ McShea (1991), p.2

${ }^{8}$ For motivations for this, and of the general reductionist approach of complexity, see part six below.
} 
interaction as feedback not imply that complexity has already been read into the interpretation from the beginning? These questions can obviously be multiplied.

The following discussion will have two parts. I will begin by exploring the limits of the intuitive concept of complexity, which I believe acts as a regulative concept for its scientific uses. This section will be primarily centered around a number of thought experiments designed to elucidate and pinpoint the core of the intuitive or everyday concept of generalized complexity. I hope to show that, via these thought experiments, that neither structural nor dynamical notions can fully explicate the concept of generalized complexity, and that in order to capture this notion we must turn to a different genus of concepts altogether. The second part of this paper will examine an example from the literature (centering on the 'robustness' of complexity), to determine whether the explanation I am proposing can adequately account for at least some of the principal properties of complexity. Finally, I will close with a few general observations concerning complexity's alleged holistic or anti-reductionist characteristics.

To begin with, let us look at some 'classical' explications of complexity. The editors of 'Emergence', an 'International Trans-disciplinary Journal of Complex Social Systems' in a preface entitled 'What is Complexity Science?' (Richardson \& Cilliers, 2001) offer the following.

The most useful definition [of complexity] that we personally have found refers to the definition of a complex system [...] which is simply given as a system that is comprised of a large number of entities that display a high level of nonlinear interactivity.

This characterization is very close to that of Simon (1962). I think we can prescind from interactivity in the above and assume that the structural concepts ('large number', 'nonlinear') carry the weight of the definition. Interactivity may mean no more than inter- 
relation, in which case the definition could be extended to static systems such as crystals, snowflakes, or mathematical entities, which at least on some accounts are genuine candidates for complexity. At minimum, the inclusion of interactivity in this definition can be understood to imply that there must at least be some sort of 'variation' or change within a complex entity, over time, whether internal to the structure or not. ${ }^{9}$ So for now, I wish to put interactivity to one side; I will return to it and similar notions further into the discussion. We are left, then, with structural notions. Yet, are structural notions enough?

\section{Complexity, Structure, and Entropy.}

Let us try to push the boundaries of the structural definition, by looking at the example of a junk heap, which according to this scheme is a complex, but not organized object $^{10}$. A car is a complex object, in that it has many components, that interact in different ways, that are involved in different relationships with one another, and so on. A junk heap (of broken cars, say) has a similar level of complexity in that the number and differentiation of parts is similar to that of a working car or collection of cars, but we hesitate to call such a junk heap organized, for such a heap does not appear to be put together in such a way that it can fulfill a function. A junk heap is disorganized, but complex. Were the junk heap to be an art installation, however, then we could impute a

\footnotetext{
${ }^{9}$ Thus, if one denies that either snowflakes or crystals are complex (e.g. Ladyman, Lambert \& Wiesner (2013), one could still claim that their formation or generation in time may very well be complex. Goldenfeld \& Kadanoff (1999) define complexity as 'structure with variations'. The extent to which even static objects can be counted as complex varies in the literature. Cilliers (1998), p.14, says that 'complex systems change with time'; Ladyman, Lambert \& Wiesner (2013), p.14, claim that 'Neither non-linear dynamics nor linear dynamics can be necessary conditions for complexity, since complexity scientists also study static structures'. Some of the proffered measures of complexity discussed in Lloyd (2001), such as difficulty of creation, would seem to be compatible with static entities.

${ }^{10}$ The example of the junk heap is from McShea (1991).
} 
purpose to it, and it would cease to appear disorganized. But purely as a junk heap, it is a complex, but disorganized object.

There is no doubt that a junk heap is disorganized, but can complexity really coexist with (a large measure of) disorganization? Is it best to think of a junk heap as complex? Is the morphological or structural account adequate in this case?

In fact, a junk heap does not seem to be the kind of thing that is usually evoked by complexity theorists as an instance of complexity. It is certainly not a paradigmatic complex object, unlike, say, a thunderstorm, a galaxy, or a biological cell. If a working car is as, or nearly as complex as, a broken-down car, why is it that the kind of thing exemplified by the working car, the organized object, is more often cited as an example of complexity than the disordered object? Decay products, or residues, or non-living or non-functioning exemplars of living and functioning beings don't seem to make the roster of complex objects as much as their organized counterparts do, despite being as complex (on the structural account).

In an essay on process philosophy, Nicholas Rescher (1996, p. 101) states that process philosophers are optimistic and tend to identify processes with 'forward' looking processes, processes that tend toward progress and improvement or complexity in some way or another, rather than with processes that tend towards the opposite side of this spectrum $^{11}$. I think a similar kind of ambivalence enters into the case of the junk heap. Structurally or morphologically, a junk heap is certainly complex, but when we examine the range of objects that are usually taken to exemplify complexity, it is in fact a certain kind of complex object that is always picked out from among the set of entities that are

\footnotetext{
${ }^{11}$ See note 16 infra.
} 
structurally complex, and junk heaps are not usually part of that roster. Intuitively, junk heaps are not complex.

Now one property that the complexity of a junk heap does not exemplify is emergence. The complexity of a junk heap does not seem to have emerged out of its components. It may indeed be a holistic property of the heap, in that it is a characteristic of the whole and not any one part, but it is not anything above the arrangements and orders of its parts, whereas the complexity of a functioning car does indeed seem to be more than this. Here is where prior states, and so temporality, may come in, for it appears that decay products in general (and a junk heap is a kind of decay product of a car or a bunch of cars) do not fit our intuitive notions of complexity because they represent the outcome of a process of entropic increase. That is, despite similar levels of organization or structure, decay products, since they are closer to states of equilibrium than products that are not the result of decay, are less complex than other products or entities that are not the result of breakdown or decay. On this account, complexity must be result of a process that emerges from a state of higher entropy. That is, a complex structure must be in a state of lower entropy (and so more ordered, in whatever sense of order is associated with far from equilibrium states) than its components. And a heap of car parts is definitely not more ordered, in this sense, than either a working car, or, say, a partially assembled but still not working car.

Some, following Poli (2013) or Cilliers (1998), might want to make a distinction here between complexity and complication. Complicated objects, being mere aggregates of components, lack the integration and holistic nature that characterize true complexity. On this account one could claim that the apparent complexity of a disorganized object 
such as a junk heap is in fact complication, not complexity, for it lacks the integration that truly complex - and by extension, organized - objects have. But is this distinction enough?

There are two possible objections to the idea that the complex-complicated distinction is helpful in isolating the meaning of complexity. First, it is not always obvious that simple (non-complex) entities lack integration. If I am correct that objects at the high end of the entropy scale are never (intuitively) complex, than the conclusion follows immediately, for the disorder measured by entropy may very well be very different from the 'dissipation' or lack of integration found in merely complicated objects. Integration, the integration that Poli and Cilliers claim characterize complex objects, is surely a purely structural property of objects, not a statistical property as is entropy. Cilliers, for instance, mentions airplanes and computers as instances of complicated, but not complex objects. Yet surely both, considered in terms of the parts that compose them, are in far from equilibrium states. Second, it strikes me that integration, or the lack of the ability of individual part-by-part description to adequately characterize the whole, is a non-analytic property, similar in this way to 'feedback' mentioned above. Whether one is able to characterize an entity as integrated or not may well hinge on one's decision to view the object as complex or complicated to begin with. Can a part by part description of a computer truly yield a full description of it, as Cilliers claims, while a comparative description of the brain cannot? Cilliers contends that the components of a complex system may shift and change, while those of a complicated system do not. But surely, considered functionally, the parts of a supposedly complicated system such as a computer do shift and change; components that store memory change 
their values, data may be stored in read-only memory or in 'virtual' memory on the hard drive, files can be stored in consecutive bites or distributed across many locations, and furthermore these paths or maps of a file's distribution in memory can themselves be stored for easier future access, perhaps rather similar to the way frequent activity in the brain is alleged to create new neural pathways. From the point of view of function, computer parts do indeed change. Likewise, jets can be upgraded, fitted with new equipment and software - computers and jets are in reality no more static than brains are. Thus, neither complexity nor complication is a transparent property; their application depends on a great deal of prior interpretation.

The connection between thermodynamics and complexity is well-known, though not necessarily well-understood. It is easily seen that it is entropy that guides our sense of complexity in a great many instances ${ }^{12}$, not structural properties, like those in the complex/complicated distinction. We can see this by looking at another example, or rather a pair of examples, that involve mixing, distribution, and equilibrium. Compare a vial of oil and water, in equilibrium, with pebbles and grains of sand along a sloping shorefront. In the first example, the fully mixed combination of oil and water appears to be more complex than the vial with the oil and water separated. Oil is lighter than water and naturally rises to the top of the vial, but we would hardly call the fully differentiated example an example of a complex arrangement. Likewise, melted and refrozen ice cream, with the milk and water components now separated, is hardly more complex than the original mixture, which will begin to separate once placed in a environment with temperature above zero Celsius. But let us now turn to the seashore. If one looks closely,

\footnotetext{
${ }^{12}$ Being in a far from equilibrium state is, according to Cilliers (1998), p.15, a necessary condition of complexity.
} 
one can see that the stones and sand, just under the point where the beach meets the water, are arranged according to size and weight, with the lighter and smaller particles further up the slope, towards the surface, and the heavier and larger pebbles further down, away from the beach. This beachside product of separation, the various pebbles and stones separated and arranged, according to size and weight, by the action of the waves, does appear to us to be more complex than unarranged sand. Contrast this to the oil and water example, where the unarranged (that is, mixed) product appears to be more complex than the unarranged sample.

Thus, structure or arrangement, by itself, is no guide to (general) complexity, for here we see that in different cases identical patterns may have opposite levels of complexity — in one case the homogenous mixture is the more complex, in the other case it is the heterogeneous mixture that has a higher level of complexity than the homogenous mixture. What we need to add, to end up with something that will let us decide whether a certain arrangement is complex or not, is the concept of entropy, that is, entropic order, (which may be different from purely surface or visual order).

In fact, our notion of structure or order itself may be dependent on this understanding of complexity; in the oil and water example, it is easy to convince oneself that the homogenous mixture is more structured than the separated mixture, because the homogenous mixture is in a far from equilibrium state. In the oil and water example, the mixed state is a state that is not in equilibrium; consequently, it is in a lower probability state, and so seems to an observer to be more ordered (and hence more complex) than the state of equilibrium where the oil and water form separate bands of liquid. If one were to encounter such a vial in its mixed state on a table in an empty room, for example, we 
would tend to conclude that either the bottle either was not a closed, isolated system as we had supposed, or that we had by chance encountered the bottle in a statistically improbable state. Either the bottle had been shaken recently, when someone had come in to the room, picked up the vial, shaken it, placed it down, and left, or that the mixed up structure had recently emerged from an unmixed state, purely by chance.

Contrast this situation with a vial of liquid in its separated state. It could have been sitting on the table in the empty room for an indefinite period; it has not emerged from anything, for there is no evidence that an hour ago, yesterday, the day before yesterday, or at any time before that the situation was any different from now. One might claim that emergence need not be understood in a temporal sense, and that the complexity that could in fact be seen in the structure of the oil and water when separated can be understood to have emerged out of the various elements of the situation at hand: the oil, the water, the vial, the presence of gravity, and so on. But surely the same could be said of the vial in its mixed up state. If we compare the two states, the order in the separated state, when we compare it with the mixed up state, now appears as too simple, too linear. The mixed up state has the correct measure of 'chaos' or non-linearity and unpredictability that is needed to make it complex. In the pebble and sand example, the opposite occurs: the mixed, homogenous state is now at equilibrium, while the separated state is in a far from equilibrium situation, and so now appears to be more ordered and complex. Here the mixed up state is purely chaotic, for there is nothing that contributes to its ordering. 


\section{Complexity Everywhere.}

There is no denying that the world we live in is complex ...

- Paul Cilliers, Complexity and Post-Modernism

Complex systems are the usual, normal case. All living systems, all psychological systems, all social systems are complex.

— Roberto Poli, A Note on the Difference Between

Complicated and Complex Social Systems

Thus, it would appear that motivations or intuitions, in this case from statistical mechanics, which are external to an object's morphology, are necessary in order to decide exactly where on the order-disorder continuum complexity lies. As we have seen, and as is often pointed out, complexity appears to lie partway between complete lack of order and the presence of too much order ${ }^{13}$. Complexity needs a measure of chaos or disorder to be truly complex. Yet structural considerations, taken apart from other notions, such as those brought in from statistical mechanics (entropy) or intentionality (in the case of functional notions such as organization) by themselves cannot tell us where along this continuum complexity is to be found. As a consequence, in areas where dynamical notions are not clearly applicable, where we cannot determine with any degree of certainty exactly what kind of order or structure is likely to constitute a state of equilibrium, or whether in fact states of equilibrium are even definable, there will be no

\footnotetext{
${ }^{13}$ For example: '... the one point of agreement in the complex systems community is that a measure of complexity should assign its highest value to systems which are neither completely random nor completely ordered.' Ladyman, Lambert and Wiesner (2013).
} 
clear guide as to where on the continuum complexity is to be placed, if it can even be placed at all, other than by making arbitrary assumptions.

To illustrate with a brief example: Imagine the dynamics of groups of people inside and outside of some sort of gathering place, such as a shopping mall. The people belong to various ethnic groups (for sake of the example). The people milling about outside we shall regard as somehow 'free' or unconstrained. As they enter the mall, they gradually disperse and become subject to various influences from within the mall, which we will regard as isolated from all other external influences. What we would like to determine is the influence of the various forces inside the mall on the population distribution according to ethnic group. There are a number of possible scenarios. In the first, people are initially distributed randomly outside; after a certain period of time in the mall, people have formed groups organized by ethnicity. People leave and join various groups in no determinate amount, so each individual group is not stable, some growing, some shrinking; however, each group nevertheless has a clear majority of one particular ethnicity at any time.

The socio-economic dynamics within the mall have transformed an undifferentiated group of people into groups organized by ethnicity (perhaps various stores cater to various ethnic groups, but the precise causes don't really matter at this point). We could say that a kind of complex pattern has emerged, a kind of grouping pattern, out of the less structured pattern outside.

But now let us look at a pattern that is the exact inverse of the above. In this case, the distribution of people outside the mall is similar to the distribution of people inside the mall in the first example, while the distribution inside the mall in this second case 
now resembles the initial configuration of the first. That is, ethnic grouping occurs in the initial state, but yields to a roughly random distribution once the forces inside the mall have had their effect. In this case, the mall dynamics have changed an ordered situation to a near random one. Still, it is possible to view this near random distribution as a complex one. One could say that the mall dynamics have broken up the ethnic-affiliated groupings found outside the mall into groupings with near equal distribution of ethnicities in a way roughly similar to the way the oil and water are distributed in the vial after being shaken up; after the infusion of market forces, a complex mixing pattern has disturbed the resting pattern of ethnic affiliations and has replaced it with a more integrated and so more complex pattern of affiliations, more complex than simple ethnic affiliation.

How can both patterns be complex? How can one particular pattern be complex in one situation, but simple in a different situation ${ }^{14}$ ?

One answer may be the following: complexity follows on intuitions from a variety of areas, such as thermodynamics, dynamical systems theory (chaos theory) or living processes, in addition of course to the structural notions mentioned above ${ }^{15}$. In certain circumstances, such as in the first two models discussed, our understanding of what makes an object complex depends on intuitions culled from our knowledge of the dynamics of the entities respective environments (the seashore in one case, a closed vial in the other). In other situations (especially in the social sciences) there may be no clear notion of entropy or evolutionary development, so whatever is able to be seen as more developed can be seen as more complex, and in the mall example, what both inside-the-

\footnotetext{
${ }^{14}$ If one wants to claim, with Poli (2013), p.145, that 'all social systems are complex', one can still investigate degrees of complexity.

${ }^{15}$ McShea (1997) conjectures that our sense of complexity in the evolution of living creatures may depend on intuitive and anthropocentric notions of hierarchy.
} 
mall distributions have in common is that both are the outcomes of a process. However, this sense of process may be guided by motivations that may have little to do with structure. For even the absence of structure can be seen as contributing to complexity, if such absence is understood as a new product of a process of some sort or another, especially, as noted in section three, a forward-looking ${ }^{16}$ process.

We must turn then, not to structure or dynamics, but to ontology; specifically, the ontology of process philosophy, for I would contend that the notion of process is able to resolve the tensions between structure and change that exist within most definitions of complexity. Complexity lies partway between order and chaos, much as process is a synthesis of old and new. According to Rescher, a process both reproduces itself, and produces novelty ${ }^{17}$. Complexity, as we have seen, always appears to exist as a kind of mediation between order producing sameness and the randomness of novelty. If we remember that processes always involve development over stages, we can see how simple repetition produces order, and novelty produces disorder, and can make the analogy between the mix of order and randomness in complexity, and the mix of novelty and identity preservation in processes rather explicit.

Let us start with a simple linear order. Of course, a linear order such as we see in the natural numbers in their conventional ordering $<1,2,3 \ldots>$ is not a simple reproduction of the first element, as in $<1,1,1 \ldots>$; however, it may be generated by the repeated application of the same rule: add 1. So in this sense such a simple arithmetical

\footnotetext{
${ }^{16}$ The question of what exactly distinguishes forward-looking processes from other kinds is as intractable as the problem of complexity itself. Rescher (1996) claims that it is a tendency towards complexity, but such an explanation obviously will not do here. As we have seen, entropy may play a role, but only where it is definable. Complexity can be found in dissipative systems, and in states with high levels of entropy (crystals). I believe this apparent interdefinability of complexity and process is telling.

${ }^{17}$ For reproduction or sameness, Rescher (1996), pp. 85, 86; novelty, ibid, pp. 77, 78, 80.
} 
order yields only sameness, as each element is in the same arithmetical 'ratio' to the next. It is not complex (and neither, a fortiori, is $<1,1,1 \ldots>$ ).

On the other hand, we can regard a 'rule' that (somehow) generates a random number as a rule that generates a completely 'new' number each time. The more complicated ${ }^{18}$ the set of generating rules is, the greater the difference is in each generated element and so the newer it is. Yet so long as this process is rule governed (with the caveat that the random number generating 'rule' above is not really a rule as such), there must be an element of sameness between successively generated elements, according to this analogy. Thus, an order such as can be seen in the prime numbers ordered according to magnitude $<2,3,5,7,11,13,17 \ldots>$ is more complex in this sense than $\langle 1,2,3, \ldots>$, despite still being a linear order (and so even a linear order can be complex!).

Of course, novelty and identity in process go beyond series of numbers, but this example allows us to see how one can construct a comparison between these aspects of process and the intermingling of the random and the ordered in complexity.

\section{Complexity, Robustness, and Process}

In order to test the hypothesis formulated here (that generalized complexity is best understood as process, which unites the structural and dynamic features of complexity), I

\footnotetext{
${ }^{18}$ By 'complicated' something like Kolmogorov or computational complexity can be understood. Here then is a connection between this sense of complexity and the more general one under consideration here; an important difference, however, is that while a completely random series of elements is maximally computationally complex, it is not complex in the sense discussed in this paper. The above analogy furnishes us with a reason: complexity, if always the result of a process, must always be rule governed to an extent, as a process always has a particular shape or form (for this, see Rescher (2000), p. 24 et seq.). Pure randomness is formless, and hence never rule governed. So by virtue of being a process, ordinary (non-computational) complexity cannot be random.
} 
would like to examine one of the key characteristics that Ladyman, Lambert and Wiesner (LLW) in their 'What is a Complex System?' (henceforth 'WCS') attribute to complexity, namely 'robustness' or 'robust order', which they claim is a necessary (but not sufficient $)^{19}$ condition for complexity. We will see that robust order is not only a necessary characteristic of process, but is sufficient for process as well.

According to LLW, order is robust when it is stable; that is, robust order is able to persist in the presence of perturbations. This is due to the property of being 'distributed throughout the system', as opposed to being located in some sort of particularly locatable central control. For example, a flock of seagulls is able to stay together despite fluctuations in wind patterns or the loss of particular members of the flock. Another example given in WCS is that of a living cell. A cell can repair itself when broken. A crystal, however, cannot ${ }^{20}$. In computational terms, a robust order possesses the capacity for a certain degree of 'error correction' or the ability to self-mend ${ }^{21}$.

The first thing to note is that robustness is clearly a non-structural requirement. Robustness cannot be described in structural terms; robustness has to do with preservation of structure, not with the constitution or the composition of the structure itself. So a crystal is clearly non-robust in this sense, as it posses plenty of structure, but is liable to chipping or fragmentation and so loss of structure, and it is not obvious that

\footnotetext{
${ }^{19}$ Robustness is one of several characteristics that LLW claim are necessary, but neither individually nor jointly sufficient for complexity.

${ }^{20}$ The implication here of course is that a crystal is not complex. LLW seem undecided about this issue. Crystals are cited along with ant colonies as examples of emergent systems, although they admit that emergence is only a necessary condition for complexity. What may be missing is the presence of 'higher-level order' in the object. As an example of a non-complex object with emergent properties LLW give an ideal gas. But surely crystals (and ant colonies) posses much more order than ideal gases! In other places LLW cite crystals as examples of systems with too much order to be truly complex.

${ }^{21}$ LLW, 39-40.
} 
adding structure would somehow make it less brittle, nor that it is the presence of more complicated kinds of structure that make robust systems such as schools of fish or flocks of birds more robust. So, robustness seems to be a genuine addition to structure.

But what exactly is the nature of this additional quality or capacity? It must be noted that some kind of robustness or quality equivalent to robustness is a necessary component of any identifiable object at all, not simply complex objects. If robustness is a kind of ability to persist through change — and it appears to be — then it may be simply a kind of identity condition. Any object whatsoever that has an identity must have some sort of ability to persist through change, otherwise there would be no basis for its identity. Even a crystal, despite being subject to structure destroying flaws, has a kind of robustness in it. Many crystals can emerge from submergence in water intact (although some cannot); others can tolerate some degree of heating or cooling without loosing their structure, and all crystals can endure through at least some sort of extended passage of time (in fact most crystals are of course very long lived objects, persisting longer than the average school of fish or flock of birds!). Today's crystal is still tomorrow's crystal, and so on. In fact it would appear that, given this interpretation of robustness, crystals are in certain ways much more robust than many living objects, as they can withstand much greater extremes of temperature and pressure than any living object can. A heated crystal will, once the source of heat has been removed, return to the ambient temperature of its surroundings, and so 'self-correct' in a sense.

Is robustness then simply another word for an identity condition shared by complex and non-complex entities alike? Is robustness a trivial quality? Perhaps not, for an objection could run as follows: robustness is not just persistence though change, but a 
certain kind of persistence, the ability to correct or to reconstitute structure, not simply to preserve structure, as crystals and other non-complex objects do. A heated or immersed crystal may undergo and later recover from change, but it loses none of its structure in the process. Even the classic paradox that involves persistence through change, the boat that has all of its planks removed one by one, while still remaining the 'same' boat, looses material, but none (or little) of the arrangement or structure of its planks ${ }^{22}$. A truly robust boat would be able to put itself back together, so-to-speak, even after it had been shipwrecked or otherwise significantly damaged, not simply persist through accidental changes such as temporal change in a crystal or a material change of planks.

It is true that a broken crystal (or boat) cannot mend itself, while a cell can, and likewise a hurricane can regain strength after temporarily passing over land. So robustness would seem to be more than simply an identity condition. However, it must be noted that the identity conditions for substances and for processes are rather different, and if we take a look at these conditions we will find that some light is thrown on the situation.

Very roughly, a substance is individuated by its properties, while it undergoes change by modification of accidents. Under a classical Aristotelian realist understanding of substance (which may or may not be identical with that held by the historical Aristotle, but we will leave that problem aside), a substance's identity is guaranteed by its form, which is thought of as a kind of actualization of the substance's being. This being is usually defined or articulated as the possession of certain kinds of properties or fully actualized capacities. For example, an eye has the property of sightedness, and sulfur is

\footnotetext{
${ }^{22}$ There are various ways of interpreting the so-called 'Ship of Theseus' paradox, and formal identity is not the only nor necessarily the best one, but it will suffice for the example.
} 
odorless. Substances can undergo temporary kinds of change without losing their (necessary) properties, so an eye can be temporarily shut, without however becoming sightless, and sulfur can posses odor in the presence of impurities, but it itself is still (somehow) odorless (there are plenty of complications in this picture, but they can be left aside for the sake of the discussion.) The central feature for the purposes of this discussion is that properties are always somehow always to be thought of as present or actualized, in whatever manner that might occur in each case (which can vary greatly indeed - somehow we have to think of a shut eye, for example, as still sighted and not just potentially sighted, but I take it that this is possible). Hence, a substance can never lose any of its necessary properties without loosing its essential integrity and its being as this or that kind of substance; a sightless eye is no longer an eye. Molten sulfur is red, not yellow, but it is still sulfur, so from the point of view of substance we would probably have to conclude that sulfur is in fact 'really' colourless.

It is quite a different situation with regard to processes, however. In contrast with substance, a process is always coming-to-be; it is never fully actualized ${ }^{23}$. A process always possesses a certain measure of 'reserve' capacity ${ }^{24}$. We can think of a process as trading loss of stability or definiteness for looser identity requirements. So a hurricane, if thought of as a process (which does seem to be a natural way to think of a hurricane), need not always maintain a certain shape or form; it can loose its form, for it may still have a certain amount of capacity to regain this form at some later time, were it to loose it

\footnotetext{
${ }^{23}$ According to Whitehead, "nature is never complete", "it is always passing beyond itself" (quoted from Weber (2004b)).

${ }^{24}$ To say that something posses a 'reserve capacity' seems awfully un-process like. Whitehead himself was uneasy with the language of hylomorphism (Weber (2004)). Perhaps a property location language formulation would be more appropriate-instead of an object possessing a reserve capacity we could instead call it a 'locus of repetition of forms'. But in lieu of a complete exposition of process philosophy, I shall stick with conventional language.
} 
due to some disturbance. An even better way to put it is to state that its form is somehow inseparable from its (the form's) generation. So a hurricane doesn't so much as have a spiral shape, as it is in the state of having or consisting of a generation or production of a spiral shape. This is not, however, really the same as possessing a capacity for being spiral-shaped, for that too can be thought of as a kind of static property, like sightedness of an eye. Likewise a flock of birds can be dispersed (temporarily), while still remaining a flock, for the potential for turning into a flock may still somehow be present in some manner or other in some other stage of the process of the flock's existence. A process never stands still; it is always in transition from potentiality to actuality, and so may very well, indeed must, secure its identity through something other than static properties.

We can conclude that the structures of complex systems are neither accidents nor properties of that system. The particular structure of a complex system is integral to its identity and to its nature, but at the same time (and somewhat understandably given that most structure is a 'surface', and very often visual phenomenon) it is able to be disrupted or destroyed. Yet such disruption very often, if kept within certain parameters, does not lead to the destruction of the system itself. So we have a bit of a paradox ${ }^{25}$, philosophically speaking, in that we have an entity with a surface, accident-like property (the particular complex structure that characterizes the system), that may come and go without disturbing the essential nature of the system, but which is central to the system's identity. Nor is this structure dispositional like the sightedness of an eye, which may remain in place despite temporary lapses of activity. Complex structure can be emergent,

\footnotetext{
${ }^{25}$ This 'paradox' extends to other aspects of processes: J. Seibt (2004) has argued that processes can fulfill the logical functions of both tokens and types at once, though are best thought of as neither.
} 
but it does not seem helpful to think of it as latent ${ }^{26}$. So my suggestion is that complex systems are best understood as processes, which will explain the structural robustness of complexity. This conclusion can be further strengthened once we realize that robust order may not only be a necessary but in fact a sufficient condition for process; it is sufficient, so long as the order involved is an essential, necessary characteristic of the entity involved. Thus, for example, a printed pattern (in ink) on the surface of an elastic object such as a rubber ball might be considered robust, as it can recover from deformation that occurs whenever the ball is compressed (within certain physical limits) or deformed, but such a pattern is not really a necessary property of said rubber ball or of its elastic properties. It is merely an accidental form impressed on the surface of the ball. It is not, therefore, 'distributed throughout the system' in the way the structure of a flock of birds is so distributed. In other words, it is not only restricted to the physical surface of the ball, it is, more essentially, not fully integrated into the ball's physical or dispositional composition, in the way that, to chose a slightly clearer example than the ornithological flock, the velocities and positions of groups of air molecules in a confined gas are related to the energy levels or temperature of the gas. Such a pattern is, therefore, not really the outcome of a process; for similar reasons, it is not genuinely complex. Since it would appear that a substance oriented approach has central difficulties in dealing with such necessary, but temporarily variable, non-latent properties such as we have seen often characterize fully complex structures, a process-oriented approach could prove to be an ideal solution (provided of course that one accepts the validity of a process oriented

\footnotetext{
${ }^{26}$ It seems to me to be no accident that emergence is so often associated with complexity, as if in recognition that the more traditional terminology of latency or actuality and potentiality fails to capture something essential in complexity.
} 
approach in the first place, as well as the concept of a necessary but potentially transient non-latent property.)

An analogy might help. One can think of a complex system as a kind of musical performance ${ }^{27}$. Clearly more similar to a process than a static substance-like entity, it is also possesses a structure, a musical motif $f^{28}$, which is robust. Interrupted, it continues along in the same manner. It is always in the process of creating and bringing forth harmonies and melodies. If a particular sequence of chords is interrupted, it can 'repair' itself and reproduce those 'same' chords further along in the performance. Even out of a moment of silence, during a lull in the melody, the structure of the melody can emerge once again once the orchestra resumes playing. Yet it appears one cannot say that the melody was somehow latent in the period of silence, in the way that sightedness still remains in the momentarily shut eye. It is possible to identify the 'essence' of the performance with some central feature of the motif, say a recurrent chord or harmonic structure, yet this essence is neither constantly present nor somehow dispositionally latent in the remaining parts of the score.

\section{Concluding Remarks - Complexity, Process, and Anti-reductionism.}

Thus, what is the result of our search for a general definition of complexity? What connects the complexity of the order found in the vial and the pebble examples, in the

\footnotetext{
${ }^{27}$ By performance I mean here the phenomenological experience of the process, or perhaps even the musical motif considered as a purely temporally extended entity, abstracted away from the memories or capacities of the performers and their instruments. It helps the example to think of the motif as unwritten and containing much improvisation.

${ }^{28}$ To be more accurate, the principle harmonies or melodies of the musical motif in their temporal manifestation or performance.
} 
examples of population distribution inside a mall, in the robust structure of the flock of birds and in the musical performance, and furthermore, what excludes randomness from being complex? It is not, I would claim, morphological structure, nor a notion such as equilibrium, but a metaphysical or philosophical judgment; that is, the assumption that all of these examples exemplify processes ${ }^{29}$. It is therefore a mistake to think that there exists a general notion of complexity that can be revealed, or can be defined, by structure or dynamics alone. Rather, such a notion can only be grounded in ontology, the ontology of process. Complexity (in a general or univocal sense, applicable to all the examples in this essay) is thus not an empirical or scientific notion, but a philosophical presupposition, indeed a gestalt, just as McShea claims is the case for our sense of increasing complexity in evolution. Given this, it is doubtful that there can be any 'laws' of complexity, any more than there can be laws of process, beyond the general philosophical characterizations of process that can be found in Rescher and elsewhere. There may very well be domain specific universals, such as the Feigenbaum constants of chaos theory, the KAM laws of dynamical systems theory, and various quantifiable results from thermodynamics, but not for complexity simpliciter.

Finally, a few general remarks. A number of writers (Gare, 2000), Gilbert \& Sarkar, 2000) who have attempted to analyze complexity from a broader philosophical perspective, have aligned complexity theory with anti-reductionist methodologies, even to the extent of suggesting or even claiming that complexity theory is essentially nothing

\footnotetext{
${ }^{29}$ There are a number of authors who do recognize that complexity science makes normative and philosophical claims, in addition to empirical and scientific claims. See Woermann (2011), Cilliers (1998), and Gare (2000). However, the tension and ambiguity therein, the question of the extent to which a normative and philosophical approach can or cannot claim to yield quantifiable results in the natural sciences, seems to lie entirely unnoticed by these authors.
} 
but the outcome of an anti-reductionist perspective. Steven Phelan, in Phelan (2001) however, sharply disagrees with this assessment. What of the present study?

I first note that process philosophy itself admits of various interpretations that may hinge on a choice of either reductionist or anti-reductionist perspectives. For example, (Seibt, 2004, p. 116) claims that Rescher, along with other advocates of various 'classical process-ontological positions' retains the identification of individuals with particular entities, a point of view which she claims is a hangover from substanceoriented philosophies. Seibt, in other words, contends that Rescher's version of process philosophy is reductionist. Reductionism, she believes, is essential to substance-oriented philosophy (part of its 'core intuition'), and as a consequence must be alien to process philosophy. Rescher, in turn, (Weber, 2004a, p. 297) finds the issue of reductionism to be external to (i.e. distinct from) both process and substance philosophies, and so finds no difficulty with the particularism that Seibt claims that he adopts.

The question then becomes: Is the reductionist question key to defining complexity (as well as process), or is it an issue external to complexity (or external to the broader philosophical domain to which complexity belongs), as it is in so many other philosophical areas.

I would claim that one of the reasons for the comparative fruitfulness of complexity and chaos theory, compared to predecessors such as von Bertalanffy's General System Theory (GST), may be precisely its reductionism. Whereas GST dealt, necessarily, with open systems, systems that interacted with outside environments, complexity theory is able to consider comparative systems in isolation, focusing instead on internal, rather than external interaction. LLW, for instance, clearly identify internal, 
rather than external interaction, as one of their key criteria of complexity: 'The second condition [of a complex system] is for the elements of the system to have the means of interacting'. (p.58, my emphasis). The ensuing explanation makes clear that it is internal interaction among elements of the system itself that is meant.

While GST focused on what Bertalanffy called the 'equifinality' of open systems, 'the tendency towards a characteristic final state from different initial states' (Bertalanffy, 1968, p. 46) complexity science is, by contrast, primarily concerned with the identification of patterns and regularities, in other words, (as is readily apparent in most definitions of complexity), with structure. As Phelan (2001, p.132) notes, in distinguishing GST from complexity theory, 'Regularities do not exist in open systems, almost by definition.', and so 'Complexity writers who propound a holistic thesis are thus probably not complexity scientists'. Hence, in contrast to GST, where all genuine systems are open, complex systems can be considered in isolation from their environment (just as processes can be), though in fact they may very well be open to external influences. In GST, every system is, by definition, open to external influences. However, the properties studied by complexity science, such as structure, or entropy, can be, (and in the case of entropy indeed must be), considered in isolation from the external environment. So, although a flock of birds is surely connected with its environment, when considering key indicators of complexity such as robustness, internal feedback, internal interconnection and structure, the environment can safely be placed outside the analytical horizon. An open system may very well be studied by a complexity theorist, but through internal mechanisms such as internal feedback or self-regulation, that do the work that outward directed mechanisms do in GST. 
Thus, complexity theory may be said to be non-classical or non-traditional, in the same way that process theory is non-classical (compared to the substance-orientation of much of traditional philosophy and philosophy of science), yet at the same time, reductionist. Von Bertalanffy was able to articulate a materialist non-classical theory by dispensing with such non-material suppositions such as vitalism or energeticism that have proven to be incompatible with modern science (see 'The Organismic Conception' in Bertalanffy (1975), and Gilbert \& Sarkar) by accounting for the developmental properties of these suppositions in a new form ('organicism') acceptable to modern science. Similarly, complexity theory has in turn been able to adapt to modern developments in quantifiable sciences such as physics and the mathematized social sciences by jettisoning the largely holistic preoccupations of GST.

If we must reject the line of interpretation that finds complexity an outcome of a largely anti-reductionist methodology, and at the same time conclude that complexity cannot be defined in empirical terms, where does that leave us? I find that it is process, rather than holism or anti-reductionism, that best provides a philosophical perspective on complexity. As what exactly one is to make of this perspective itself, I have attempted to sketch, in Taborsky (2010), an analysis of both complexity and process as articulations of a particular kind of ontology, an ontology of multiple or unbounded causation, as opposed to the bounded or one-to one causal model of classical or traditional physics. Such speculations are beyond the scope of this paper, but I note that causal, rather than entity holism, may prove fruitful in both finding common ground between process philosophy, complexity science and GST, (as claimed by many researchers such as Gare, 
Abraham, and Castellani), while allowing for fundamental differences between the latter two, as noted by Phelan.

\section{References}

Abraham, Ralph H. (2011). The genesis of complexity. World Futures: The Journal of Global Education, 67, 380-394.

Bertalanffy, L. von. (1968). General system theory. New York: George Brazilier

Bertalanffy, L. von. (1975). Perspectives on general system theory. New York: George Brazilier

Castellani, Brian. (2013). Complexity map. Art \& Science Factory.

http://www.art-sciencefactory.com/complexity-map_feb09.html. (Accessed 19 May 2014)

Cilliers, P. (1998). Complexity and post-modernism. London \& New York: Routledge

Gare, A. (2000). Systems theory and complexity. Democracy \& Nature, 6, 327-339.

Gilbert, S. \& Sarkar, S. (2000). Embracing complexity. Developmental Dynamics, 219, 1-9.

Goldenfeld, N. \& Kadanoff, L. P. (1999). Simple lessons from complexity. Science, 284, 87-89.

Kuhn, T. (1987). What are scientific revolutions? In L. Krüger, L.J. Daston \& M. Heidelberger (Eds.), The probabilistic revolution: Volume 1: Ideas in history (pp.7-22). Cambridge: The MIT Press.

Ladyman, J., Lambert, J. \& Wiesner, K. (2013). What is a complex system? European Journal for the Philosophy of Science, 3, 33-67.

Lloyd, S. (2001). Measures of complexity: A non-exhaustive list. Control Systems Magazine, 21, 4.

McShea, W. (1991). Complexity and evolution: What everybody knows. Biology and Philosophy, $6,303-324$.

McShea, W. (1997). Complexity in evolution: A skeptical assessment. Philosophica, 59, 79-112.

Phelan, S. (2001). What is complexity science, really? Emergence, 3, 120-136.

Poli, R. (2013). A note on the difference between complicated and complex social systems, Cadmus, 2, 142-147.

Rescher, N. (1996). Process philosophy: An introduction to process metaphysics. Bloomington: State University of New York Press. 
Rescher, N. (2000). Process philosophy: A survey of basic issues. Pittsburg: University of Pennsylvania Press.

Rescher, N. (2004). Replies. In M. Weber (2004a), 289-322.

Riche, J. (2004). Process logic and epistemology, in M. Weber (2004a), 173-196.

Richardson, K., \& Cilliers, P. (2001). Special editors' introduction: What is complexity science? A view from different directions. Emergence, 3, 5-23.

Seibt, J. (2004). Process and particulars, in M. Weber (2004a), 113-134.

Simon, H. A. (1962). The architecture of complexity. Proceedings of the American Philosophical Society, 106, 467-482.

Taborsky, P. (2010). The logic of cultures: Three structures of philosophical thought. Bern: Peter Lang.

Weber, M. (Ed.) (2004a). After Whitehead: Rescher on process metaphysics. Frankfurt: Ontos

Weber, M. (2004b). Process metaphysics in context, in M. Weber (2004)a, 41-76.

Woermann, M. (2011). What is complexity theory?: Features and implications. Systems Engineering Newsletter, 30, 1-8.

Worrall, J. (2003). Normal science and dogmatism, paradigms and progress: Kuhn 'versus' Popper and Lakatos, in T. Nickles (Ed.). Thomas Kuhn. Cambridge: Cambridge University Press. 